\title{
Statistical Properties of Shocks in Burgers Turbulence, II: Tail Probabilities for Velocities, Shock-Strengths and Rarefaction Intervals
}

\author{
Marco Avellaneda \\ Courant Institute of Mathematical Sciences, New York University, New York, NY 10012, USA
}

Received: 8 November 1993

\begin{abstract}
This paper studies the structure of the random "sawtooth" profile corresponding to the solution of the inviscid Burgers equation with white-noise initial data. This function consists of a countable sequence of rarefaction waves separated by shocks. We are concerned here with calculating the probabilities of rare events associated with the occurrence of very large values of the normalized velocity, shock-strength and rarefaction intervals. We find that these quantities have tail probabilities of the form $\exp \left\{-C x^{3}\right\}, x \gg 1$. This "cubic exponential" decay of probabilities was conjectured in the companion paper [1]. The calculations are done using a representation of the shock-strength and length of rarefaction intervals in terms of the statistics of certain conditional diffusion processes.
\end{abstract}

\section{Introduction}

This paper is a sequel to a previous article [1] concerning the structure of statistical solutions of Burgers' equation with random initial data (Burgers Turbulence)

$$
\left\{\begin{array}{l}
\frac{\partial u(x, t)}{\partial t}+\frac{\partial}{\partial x}\left(\frac{u(x, t)^{2}}{2}\right)=0 \\
u(x, t=0)=u_{0}(x)=\text { Gaussian white noise }
\end{array}\right.
$$

This nonlinear wave equation is understood as the inviscid limit $(v \rightarrow 0)$ of the equation

$$
\frac{\partial u(x, t)}{\partial t}+\frac{\partial}{\partial x}\left(\frac{u(x, t)^{2}}{2}\right)=v \frac{\partial^{2} u(x, t)}{\partial x^{2}} .
$$

The statistical properties of Burgers Turbulence have been widely studied since the original works of Burgers [2] and Hopf [3]. In particular, expectation values, moments and correlation functions of the velocity, $u(x, t)$, have been calculated. A modern and interesting account of the theory of Burgers Turbulence and other related systems, as well as of its role in recent cosmological theories, can be found in Gurbatov, Malakhoff and Saichev [4]; see also [12]. 
Let us review briefly some known properties of statistical solutions of (1) which are relevant to this paper. The initial velocity $u_{0}(x)$ can be viewed as a highly oscillatory function that is spatially uncorrelated and assumes the values $+\infty$ and $-\infty$ everywhere. The integral of the initial velocity is statistically a Brownian motion, or continuous-time random walk. At positive times, the solution evolves according to the hyperbolic equation in (1), with shocks forming and interacting with each other. When two shocks travelling at different speeds overcome each other, they merge into a larger shock which travels at a new speed, determined by the Rankine-Hugoniot condition [5]. Interacting shocks behave like particles undergoing inelastic collisions, in which mass and momentum are conserved (but not energy, which is dissipated in the collision). The solution of (1) at positive times is a piecewise linear random function, with a countable, discrete, set of jump discontinuities corresponding to shocks [1]. The piecewise linear portions between consecutive shocks have the same slope $1 / t$ and correspond to rarefaction waves. The velocity profile can therefore be seen as a statistically homogeneous "sawtooth" function formed by a sequence of consecutive random shocks followed by rarefaction waves ("N-waves").

Due to the self-similarity of the initial datum $u_{0}(x)$, the velocity satisfies the scaling relation $[1,7]$

$$
u(x, t) \approx \frac{1}{t^{1 / 3}} u\left(\frac{x}{t^{2 / 3}}, 1\right)
$$

where " $\approx$ " denotes statistical equivalence. Therefore, the spatial structure of Burgers Turbulence at arbitrary times can be deduced from the solution at time $t=1$ by rescaling. This reflects the fact that the system (1) corresponds to the long-time, large-scale limit of the viscous equation (2). In this limit, all times become infinitely large with respect to the diffusion timescale and hence are equivalent after a similarity transformation.

The goal of this paper is to complete the study initiated in [1] which gave various results on the probability distribution of the velocity, shock-strengths and lengths of rarefaction intervals of the function $u(x, t)$. Unlike other works on Burgers Turbulence, such as [2, 3, 4], this paper and its predecessor [1] focus on extreme properties of these distributions, such as the probability that a given shock has very small or very large intensity. The motivation for this investigation comes from the numerical works of Kida [6] and She, Aurell and Frisch [7], who studied the statistics of very small shocks. A rigorous proof of the scaling laws proposed in these papers was given in [1]. A different kind of initial datum was studied rigorously by Sinai [8], also in the context of small shock-strengths. Here, we concentrate on the opposite asymptotics, i.e. on the probability of occurrence of very large shocks.

The scaling form of the distribution for extremely large values of the shock strength was conjectured in [1], where it was shown that the tails of the velocity, shock-strength and rarefaction interval distributions could be bounded from above by a cubic exponential function $\exp \left\{-C x^{3}\right\}$. Notice that when applied to the velocity $u(x, 1)$, this bound implies that the velocity PDF is non-Gaussian and has very short tails. In this article, we shall establish rigorously that the cubic exponential scaling function gives the correct asymptotics for the three quantities. These results give us a more complete picture on the structure of Burgers Turbulence.

Our main objective is to prove

Theorem 1. Let $u(x)=u(x, 1)$ be the solution of (1) at time $t=1$, let $S$ represent the strength of the first shock to the left of $x=0$ and let $\delta x$ represent the 
length of the rarefaction interval containing $x=0$. There exist positive numerical constants $C_{1}$ and $C_{2}$ such that for all $u \geqq 1, s \geqq 1$ and $x \geqq 1$,

$$
\begin{gathered}
\exp \left\{-C_{1} u^{3}\right\} \leqq \text { Prob. }\{u(x)>u\} \leqq \exp \left\{-C_{2} u^{3}\right\} \\
\exp \left\{-C_{1} s^{3}\right\} \leqq \text { Prob. }\{S>s\} \leqq \exp \left\{-C_{2} s^{3}\right\}
\end{gathered}
$$

and

$$
\exp \left\{-C_{1} x^{3}\right\} \leqq \text { Prob. }\{\delta x>x\} \leqq \exp \left\{-C_{2} x^{3}\right\}
$$

The upper bounds in (5), (6) and (7) were derived in Theorem 3 of the companion paper [1]. Our purpose here is thus to prove the corresponding lower bounds, thereby establishing that the cubic exponential scaling form is sharp. We have not attempted to keep track of the exact values of the constants $C_{1}$ and $C_{2}$, leaving open the possibility that $C_{1}=C_{2}$, i.e. that the bounds give the exact asymptotic behavior of the probabilities on a logarithmic scale. However, we believe that more refined analysis along the lines of this paper is likely to give quantitative information on the constants as well.

The mathematical approach for deriving the estimates is based on the study of rare events for certain diffusion processes associated with Burgers Turbulence. These processes arise naturally by considering the variational solution of the HamiltonJacobi (eikonal) equation satisfied by the velocity potential; cf. equation (8). With our approach, the statistical quantities of interest can be described in terms of the absolute minimum value and the time at which the absolute minimum is attained for random processes conditioned on never hitting a linear boundary. This description of the shocks and rarefaction intervals was introduced in [1], where the connection between the statistics of Burgers Turbulence and those of the convex hull of Brownian paths, first studied by Groenboom [9] and Pitman [10], was noticed. In that first paper, we used this technique to calculate the statistics of small shocks. It turns out that a characterization of the probabilities for large values of shocks, etc. can also be made using conditional diffusions. Roughly speaking, the reason for the cubic exponential scaling is that the tail probabilities can be determined from steepest descent, or Laplace-type asymptotics, applied to either standard Brownian paths or conditioned diffusion paths, depending on the quantity of interest. For example, a large value of the velocity $u(x, 1)$ corresponds to a Brownian trajectory $\beta(\cdot)$ satisfying $\beta(u)<-C u^{2}$, for $u \gg 1$, an event with probability of the order of $\exp \left\{-C u^{3}\right\}$. The arguments for shock-strengths and rarefaction intervals are similar in spirit but require the use of conditional diffusions and hence are of a more technical nature.

This paper is organized as follows: in Sect. 2 we discuss the tails of the distribution of the velocity $u(x)$, where $x$ is an arbitrary real number. Here, consideration of standard (free) Brownian motion is sufficient for our purposes. The lower bound is obtained by choosing an "event" in the space of Brownian trajectories (associated with a set of initial data $u_{0}$ ) on which the magnitude of the velocity at the origin $x=0$ is necessarily larger than a specified large constant $u$. We then show that such event, or set of realizations of $u_{0}$, has probability at least $\exp \left\{-C_{1} u^{3}\right\}$. In Sect. 3, we turn to the study of the tails of the distribution of shock strengths. Again, we find an event in the ensemble of initial velocity configurations which 
has the correct probability $\left(\geqq \exp \left\{-C s^{3}\right\}\right)$ and on which the shock-strength $S$ exceeds a large constant $s$. Unlike the case of Sect. 2, the technique for calculating the probability of such event relies on properties of a conditional diffusion process introduced in the companion paper [1]. In Sect. 4 we use a similar approach to study the rare events for the length of a rarefaction interval.

Although this paper tries to be self-contained in its exposition, it borrows several results from [1]. Therefore, a reading of the relevant sections of [1] is recommended.

\section{Tails of the Velocity Probability Distribution}

Let us recall some elementary facts about Burgers equation with random initial data. The solution of (1) can be expressed in terms of a variational principle. This is a consequence of the classical Hopf-Cole transformation linearizing (1) and (2). Define $\Psi(x, t)=\int_{0}^{x} u\left(x^{\prime}, t\right) d x^{\prime}, \Psi(x)=\Psi(x, 1)$ and $u(x)=u(x, 1)$. Then, we have

$$
\Psi(x, t)=\operatorname{Inf}_{y}\left[\beta(y)+\frac{(x-y)^{2}}{2 t}\right],
$$

and hence

$$
\begin{aligned}
u(x) & =x-\frac{d}{d x} \sup _{y}\left[x y-(1 / 2) y^{2}-\beta(y)\right] \\
& \equiv x-y(x) .
\end{aligned}
$$

Here $\beta(y),-\infty<y<+\infty$, is a two-sided Brownian motion and $y(x)$ is the point where the minimum of $(8)$ is achieved. The function $y(x)$ can be interpreted as the inverse of the derivative of the convex envelope of the function $F(y)=$ $\frac{1}{2} y^{2}+\beta(y)$, cf. [1].

From (8), (9), we know that

$$
u(0)=-y(0)
$$

where $y(0)$ is the point where $F(y)$ attains is minimum. We seek a lower bound on the probability

$$
\begin{aligned}
\operatorname{Pr} .\{|y(0)|>u\} & =2 \operatorname{Pr} .\{y(0)>u\} \\
& =\operatorname{Pr} .\left\{\inf _{y>0} F(y) \text { is attained at some }>u\right\} .
\end{aligned}
$$

Let $a>0$ be a given number. If a Brownian path $\beta(y), y>0$, is such that

$$
\frac{1}{2} y^{2}+\beta(y)>-a \text { for } y<u
$$

and

$$
2 u^{2}+\beta(2 u)<-(a+1),
$$


then $F(2 u)<\inf _{y<u} F(y)$, and hence the minimum of $F(y)$ is necessarily attained at some $y>u$. We have therefore

$$
\begin{aligned}
& \operatorname{Pr} .\{|u(0)|>u\} \geqq \operatorname{Pr} .\left\{\inf _{y \leqq u} F(y)>-a ; 2 u^{2}+\beta(2 u)<-(a+1)\right\} \\
& =\int_{-\left(a+(1 / 2) u^{2}\right)}^{\infty} \operatorname{Pr} \cdot\left\{\inf _{y<u} F(y)>-a ; \beta(u)=z\right\} \\
& \times \operatorname{Pr} .\left\{\beta(2 u)+2 u^{2}<-(a+1) \mid \beta(u)=z\right\} d z \\
& >\int_{-\left(a+(1 / 2) u^{2}\right)}^{u^{2}-a} \operatorname{Pr} .\left\{\inf _{y<u} F(y)>-a ; \beta(u)=z\right\} \\
& \times \operatorname{Pr} .\left\{\beta(2 u)-\beta(u)<-(a+1)-2 u^{2}-z\right\} d z \\
& \geqq \int_{-\left(a+(1 / 2) u^{2}\right)}^{u^{2}-a} \operatorname{Pr} .\left\{\inf _{y<u} F(y)>-a ; \beta(u)=z\right\} \\
& \times \quad \int_{-\infty}^{-\left(1+3 u^{2}\right)} \exp \left\{-\left(z^{2} / 2 u\right)\right\} \frac{d z}{\sqrt{2 \pi u}} \\
& \geqq C_{1} \int_{-\left(a+(1 / 2) u^{2}\right)}^{\infty} \operatorname{Pr} .\left\{\inf _{y<u} F(y)>-a ; \beta(u)=z\right\} \\
& \times \exp \left\{-\frac{1}{2 u}\left(1+3 u^{2}\right)^{2}\right\} d z \\
& \geqq C_{1} \exp \left\{-\left(9 u^{3} / 2\right)\right\} \operatorname{Pr} .\left\{\inf _{y<u} F(y)>-a\right\} \\
& \geqq C_{1} \exp \left\{-\left(9 u^{3} / 2\right)\right\} \operatorname{Pr} .\left\{\inf _{y<+\infty} F(y)>-a\right\} \text {. }
\end{aligned}
$$

Here $C_{1}$ is a numerical constant. Since the infimum of $F(y)$ is finite, the latter probability is positive and independent of $u$. (Note also that this probability approaches 1 as $a \rightarrow+\infty$.) Therefore, we conclude that, for a suitable constant $C>0$ and all $u \geqq 1$, we have

$$
\operatorname{Pr} .\left\{\mid u(0 \mid>u\} \geqq \exp \left\{-C u^{3}\right\}\right.
$$

This proves the lower bound in (5).

We recall, for the sake of completeness, how the corresponding upper bound was proved in [1], Sect. 5. There, an upper bound of cubic exponential type was obtained by estimating the probability that $F(y)<0$ for some $y>u$. This event must necessarily occur if $y(0)>u$, since $F(y(0))$ is always negative. The probability that $F(y)<0$ for some $y>u$ is found to be of the order of $\exp \left\{-C u^{3}\right\}$, by a direct calculation with the Gaussian distribution. 


\section{Tails of the Shock-Strength Probability Distribution}

In this section, we shall use the technique of conditional diffusions and time-reversal to study the statistics of the shock-strength ; cf. [1]. Let $S$ denote the strength of, say, the first shock to the right of $x=0$. We seek a lower bound on

$$
\operatorname{Pr} .\{S>s\}
$$

for $s \gg 1$. For this purpose, we use Lemma 4 in [1], which establishes a connection between the distributions of $S$ and $t_{u(0)}^{*}$, which we define as the time at which the stochastic process

$$
\left\{\begin{array}{l}
\widetilde{X}(t)=\beta(t)+\frac{1}{2 t}-u(0), \text { conditional } \\
\text { on } \widetilde{X}(t)>0 \text { for all } t>0
\end{array}\right.
$$

attains its minimum. More precisely, we have

$$
\operatorname{Pr} .\{S>s\}=E\left\{\mathrm{P}\left[t_{u(0)}^{*}<\frac{1}{s}\right]\right\},
$$

where $P$ denotes the distribution of $\widetilde{X}(t)$ given $u(0)$ and $E[\cdot]$ represents expectation value with respect to the distribution of $u(0)$. In other words, the random variables $S$ and $1 / t^{*}$ have the same distribution conditionally on $u(0)$. Let $t=1 / s$. Then, if

$$
\begin{gathered}
\frac{2}{t}<\widetilde{X}(t / 2)<\frac{3}{t}, \\
\frac{4}{t}<\widetilde{X}(t)<\frac{5}{t}
\end{gathered}
$$

and

$$
\widetilde{X}\left(t^{\prime}\right)>\frac{3}{t}, \quad t^{\prime}>t
$$

the minimum of $\widetilde{X}(s), 0<s<+\infty$, must necessarily occur for $s=t_{u(0)}^{*}<t$. Hence,

$$
\begin{aligned}
& E\left\{\mathrm{P}\left[t_{u(0)}^{*}<t\right]\right\} \\
& \geqq \operatorname{Pr} .\left\{\frac{2}{t}<\widetilde{X}(t / 2)<\frac{3}{t} ; \frac{4}{t}<\widetilde{X}(t)<\frac{5}{t} ; \widetilde{X}\left(t^{\prime}\right)>\frac{3}{t}, t^{\prime}>t\right\} \\
& \quad=\operatorname{Pr} .\left\{\frac{2}{t}<\widetilde{X}(t / 2)<\frac{3}{t} ; \frac{4}{t}<\widetilde{X}(t)<\frac{5}{t} ; P_{\widetilde{X}(t), t}\left[\widetilde{X}\left(t^{\prime}\right)<\frac{3}{t}, t^{\prime}>t\right]\right\} .
\end{aligned}
$$

In order to estimate the last probability, we make use of a comparison principle, Proposition 7 in the companion paper [1], which gives a lower bound on the probability inside the brackets in terms of the statistics of conditional Brownian motion (conditioned on never hitting $\beta=0$; cf. [11] or [1]). Since $\frac{4}{t}<\widetilde{X}(t)<\frac{5}{t}$, 
we have $\widetilde{X}(t)>\frac{1}{t}$ and Proposition 7 of [1] can be applied. We have, accordingly,

$$
\begin{aligned}
\mathrm{P}_{\widetilde{X}(t), t} & {\left[\widetilde{X}\left(t^{\prime}\right)>\frac{3}{t}, t^{\prime}>t\right] } \\
& \geqq \frac{1}{2} \mathrm{P}\left[\widetilde{\beta}_{\widetilde{X}(t), t}\left(t^{\prime}\right)+\frac{1}{2 t^{\prime}}-\frac{1}{2 t}>\frac{3}{t}, t^{\prime}>t\right] \\
& \geqq \frac{1}{2} \mathrm{P}\left[\widetilde{\beta}_{\widetilde{X}(t), t}\left(t^{\prime}\right)>\frac{3.5}{t}, t^{\prime}>t\right] \\
& =\frac{1}{2} \cdot \frac{\widetilde{X}(t)-\frac{3.5}{t}}{\widetilde{X}(t)} \\
& =\frac{1}{2} \frac{\frac{4}{t}-\frac{3.5}{t}}{\frac{5}{t}}=\frac{1}{20} .
\end{aligned}
$$

Here, $\widetilde{\beta}_{x, t}(t)=$ Brownian motion conditional on not hitting 0 , starting at $x$ at time $t$. In the last estimate, we made use of the fact that the infimum of this conditional Brownian motion is uniformly distributed on the interval $[0, x]$; cf. [11].

We conclude that

$$
\begin{aligned}
E & \left\{\mathrm{P}\left[t_{u(0)}^{*}<t\right]\right\} \\
& \geqq \frac{1}{20} \operatorname{Pr} .\left\{\frac{2}{t}<\widetilde{X}(t / 2)<\frac{3}{t} ; \frac{4}{t}<\widetilde{X}(t)<\frac{5}{t}\right\} \\
& \geqq \frac{1}{20} E\left\{\frac{2}{t}<\widetilde{X}(t / 2)<\frac{3}{t} ; \mathrm{P}\left[\frac{4}{t}<\widetilde{\beta}_{\widetilde{X}(t / 2), t / 2}(t)+\frac{1}{2 t}-\frac{1}{t}<\frac{5}{t}\right]\right\},
\end{aligned}
$$

where Proposition 7 of [1] was applied again, using that $X(t / 2)>\frac{2}{t}$. the probability inside the last expectation value satisfies

$$
\begin{aligned}
\mathrm{P} & {\left[\frac{4}{t}<\widetilde{\beta}_{\widetilde{X}(t / 2), t / 2}(t)-\frac{1}{2 t}<\frac{5}{t}\right] } \\
& =\mathrm{P}\left[\frac{4.5}{t}<\widetilde{\beta}_{\widetilde{X}(t / 2), t / 2}(t)<\frac{5.5}{t}\right] \\
& =\mathrm{P}\left[\frac{4.5}{t}<|\mathbf{W}(t / 2)|<\frac{5.5}{t}\right],
\end{aligned}
$$

where $|\mathbf{W}(\cdot)|$ is a three-dimensional Brownian motion starting on the sphere of radius $|\widetilde{X}(t / 2)|$ at time $t=0$ and $|\mathbf{W}(\cdot)|=\sqrt{W_{1}^{2}(\cdot)+W_{2}^{2}(\cdot)+W_{3}^{2}(\cdot)}$ (cf. [11] or [1]).

An elementary estimate based on the fact that $\mathbf{W}(\cdot)$ has multivariate Gaussian probability density with variance $t$ gives, for $t \leqq 1$,

$$
\mathrm{P}\left[\frac{4.5}{t}<|\mathbf{W}(t)|<\frac{5.5}{t}\right] \geqq \exp \left\{-\frac{C}{t^{3}}\right\},
$$

where $C$ is a numerical constant that we do not evaluate explicitly. Hence

$$
E\left\{\mathrm{P}\left[t_{u(0)}^{*}<t\right]\right\} \geqq \frac{1}{20} \exp \left\{-\frac{C}{t^{3}}\right\} \times \operatorname{Pr} .\left\{\frac{2}{t}<\tilde{X}(t / 2)<\frac{3}{t}\right\} .
$$


It remains to estimate from below

$$
\operatorname{Pr} .\left\{\frac{2}{t}<\tilde{X}(t / 2)<\frac{3}{t}\right\}=\operatorname{Pr} .\{1<(t / 2) \tilde{X}(t / 2)<3 / 2\} .
$$

For this, we shall use the definition of the process $\widetilde{X}(t)$, given in (10). Note that the corresponding unconditional process, $X(t)=\beta(t)+\frac{1}{2 t}-u(0)$, satisfies

$$
t X(t)=t \beta(t)+\frac{1}{2}-u(0) t, \quad t>0 .
$$

Introducing $\tau=t^{3}$ as a new "time" variable, we have

$$
t X(t)=\hat{\beta}(\tau)+\frac{1}{2}-u(0) \tau^{1 / 3}, \quad \tau>0,
$$

where $\hat{\beta}(\tau)=t \beta(t)$ defines a new Brownian motion, as a consequence of the statistical self-similarity of Brownian paths. From (16), we conclude that the process $t X(t)$, conditioned on never hitting 0 , is statistically equivalent to $\widetilde{Y}(\tau)$, where

$$
\left\{\begin{array}{l}
\tilde{Y}(\tau)=\hat{\beta}(\tau)+\frac{1}{2}-u(0) \tau^{1 / 3}, \quad \tau>0, \\
\text { conditioned on never hitting } \widetilde{Y}=0 .
\end{array}\right.
$$

Before giving the rigorous proof of the estimate for the tails of $S$, let us give an intuitive idea of why it should hold. Notice that $\widetilde{Y}(\tau)$ tends to $1 / 2$ as $\tau \rightarrow 0$. Therefore, for very small values of $\tau$, the distribution of $\widetilde{Y}(\tau)$ should be approximately equal to the distribution of the unconditioned process

$$
\beta(\tau)+\frac{1}{2}-u(0) \tau^{1 / 3} \cong \beta(\tau)+\frac{1}{2}, \quad \tau \ll 1
$$

This approximation leads to

$$
\begin{aligned}
\operatorname{Pr}\left\{\frac{2}{t}<\tilde{X}\left(\frac{t}{2}\right)<\frac{3}{t}\right\} & =\operatorname{Pr} .\{1<\tilde{Y}(\tau)<3 / 2\}, \quad\left(\tau=(t / 2)^{3}\right) \\
& \cong \operatorname{Pr} .\{1 / 2<\beta(\tau)<1\} \\
& \cong \exp \left\{-\frac{8}{\tau}\right\}, \quad \tau \ll 1 \\
& \cong \exp \left\{-\frac{C}{t^{3}}\right\}, \quad \ll 1,
\end{aligned}
$$

which is a "cubic exponential" function of $1 / t$. Putting this together with (15) we obtain the desired lower bound on the probability of large shocks of setting $t=1 / \mathrm{s}$.

In order to make the above argument fully rigorous, we shall use a technical lemma which is proved in the Appendix. The statement of the lemma is stronger than what is needed to estimate the probability in (16). The reason for this is that we shall make use of the full result of the lemma in Sect. 4, when estimating the probabilities of large rarefaction intervals. 
Lemma 2. Let $a, b, c$ be positive numbers such that $a<1 / 2<b<c$. Then, for all $u_{0}>0$ there exist constants $T_{0}=T_{0}\left(a, b, c, u_{0}\right)>0$ and $C_{0}=C_{0}\left(a, b, c, u_{0}\right)$ $>0$ such that for all $\tau \leqq T_{0}$, and all $u$ such that $u_{0}<u(0)<2 u_{0}$, we have

$$
\operatorname{Pr}\left\{\inf _{\tau^{\prime}<\tau} \tilde{Y}\left(\tau^{\prime}\right)>a, b<\tilde{Y}(\tau)<c\right\} \geqq \exp \left\{-C_{0} / \tau\right\} .
$$

Applying this lemma with $a=1 / 3, b=1$ and $c=3 / 2$, we obtain, for $u_{0}<$ $u(0)<2 u_{0},(t / 2)^{3} \leqq T_{0}$ and $\tau=(t / 2)^{3}$,

$$
\begin{aligned}
& \mathrm{P}\left\{\frac{2}{t}<\tilde{X}\left(\frac{t}{2}\right)<\frac{3}{t}\right\}=\mathrm{P}\{1<\widetilde{Y}(\tau)<3 / 2\} \\
& \geqq \mathrm{P}\left\{\inf _{\tau^{\prime} \leqq \tau} \widetilde{Y}\left(\tau^{\prime}\right)>1 / 3,1<\widetilde{Y}(\tau)<3 / 2\right\} \\
& \geqq \exp \left\{-C_{0} / \tau\right\}=\exp \left\{-8 C_{0} / t^{3}\right\} .
\end{aligned}
$$

Therefore, putting together (15) and (20), we conclude that

$$
\begin{aligned}
E & \left\{\mathrm{P}\left[t_{u(0)}^{*}<t\right]\right\} \geqq \frac{1}{20} \cdot \exp \left\{-C / t^{3}\right\} \cdot \operatorname{Pr} \cdot\left\{\frac{2}{t}<\tilde{X}\left(\frac{t}{2}\right)<\frac{3}{t}\right\} \\
& \geqq \frac{1}{20} \cdot \exp \left\{-C / t^{3}\right\} \cdot E\left\{\mathrm{P}\left[\frac{2}{t}<\widetilde{X}\left(\frac{t}{2}\right)<\frac{3}{t}\right] ; u_{0}<u(0)<2 u_{0}\right\} \\
& \geqq \frac{1}{20} \cdot \exp \left\{-C / t^{3}\right\} \cdot \exp \left\{-8 C_{0} / t^{3}\right\} \cdot \operatorname{Pr} \cdot\left\{u_{0}<u(0)<2 u_{0}\right\},
\end{aligned}
$$

for sufficiently small values of $t$. Since the last probability is positive, this implies that

$$
E\left\{\mathrm{P}\left[t_{u(0)}^{*}<t\right]\right\} \geqq \exp \left\{-C^{\prime} / t^{3}\right\}, \quad t \leqq 1,
$$

for a suitable numerical constant $C^{\prime}$. Finally, changing back to the variable $s=1 / t$, we have

$$
\operatorname{Pr} .\{S>s\} \geqq \exp \left\{-C^{\prime} s^{3}\right\}
$$

which is the lower bound that we want. This completes the proof of the result on the tail probabilities of shock strengths.

\section{Tails of the Probability Distribution of the Length of a Rarefaction Interval}

In this section, we prove the lower bound in (7) for the distribution of the length of rarefaction intervals of the function $u(x)=u(x, 1)$. To fix ideas, let $\left[x_{0}, x_{1}\right]$ be the rarefaction interval containing the origin $x=0$. We denote its length by $\delta x=x_{1}-x_{0}$. According to Lemma 4 in [1], we know that, given $u\left(x_{0}\right)$,

$$
\delta x=\inf _{t>0} \tilde{X}(t)
$$


where $\widetilde{X}(t)$ is the conditional process defined in Eq. (10). Let $x$ be a positive number and set $\widetilde{t}=\frac{1}{3 x}$. If the path $\widetilde{X}(t)$ satisfies the conditions

$$
\begin{gathered}
\frac{1}{3}<t \widetilde{X}(t) \quad \text { for } t \leqq \widetilde{t}, \\
1 \leqq \widetilde{t} \widetilde{X}(\widetilde{t}) \leqq 2,
\end{gathered}
$$

and

$$
\widetilde{X}(t)>\frac{1}{3 \widetilde{t}}=x \quad \text { for } t>\widetilde{t},
$$

then, since (21) implies

$$
\widetilde{X}(t)>\frac{1}{3 \widetilde{t}}=x \quad \text { for } t \leqq \widetilde{t},
$$

the path $\widetilde{X}(t)$ will be everywhere greater than $x$. Therefore, conditionally on the value of the random variable $u\left(x_{0}\right)$, we have

$$
\begin{aligned}
& \mathrm{P}\{\widetilde{X}(t)>x\} \\
& \geqq \mathrm{P}\left\{\frac{1}{3} \leqq \inf _{t \leqq \tilde{t}}(t \widetilde{X}(t)) ; \frac{1}{\tilde{t}} \leqq \widetilde{X}(\widetilde{t}) \leqq \frac{2}{\widetilde{t}} ; \inf _{t>\tilde{t}} \widetilde{X}(t)>\frac{1}{3 \widetilde{t}}\right\} \\
& =\mathrm{P}\left\{\frac{1}{3} \leqq \inf _{t \leqq \tilde{t}}(t \widetilde{X}(t)) ; \frac{1}{\widetilde{t}} \leqq \widetilde{X}(\widetilde{t}) \leqq \frac{2}{\widetilde{t}} ; \mathrm{P}_{\tilde{X}(\tilde{t}), \tilde{t}}\left[\inf _{t>\tilde{t}} \widetilde{X}(t)>\frac{1}{3 \widetilde{t}}\right]\right\} \text {. }
\end{aligned}
$$

For $\frac{1}{\hat{t}}<\tilde{X}(\tilde{t})<\frac{2}{\hat{t}}$, we can use Proposition 7 in [1], which relates the distributions of $\widetilde{X}$ and of conditional Brownian motion, and obtain the bound

$$
\begin{aligned}
\mathrm{P}_{\tilde{X}(\tilde{t}), \tilde{t}} & {\left[\inf _{t>\tilde{t}} \tilde{X}(t)>\frac{1}{3 \tilde{t}}\right] } \\
& \geqq \frac{1}{2} \cdot \operatorname{Pr}\left\{\left\{\widetilde{\beta}_{\tilde{X}(\tilde{t}), \tilde{t}}(t)+\frac{1}{2 t}-\frac{1}{2 \tilde{t}}>\frac{1}{3 \tilde{t}}, t>\tilde{t}\right\}\right. \\
& \geqq \frac{1}{2} \cdot \operatorname{Pr}\left\{\left\{\widetilde{\beta}_{\tilde{X}(\tilde{t}), \tilde{t}}(t)>\frac{5}{6 \tilde{t}}, t>\tilde{t}\right\}\right. \\
& =\frac{1}{2} \frac{\tilde{X}(\tilde{t})-5 / 6 \tilde{t}}{\tilde{X}(\tilde{t})} \\
& \geqq \frac{1}{2} \cdot \frac{1 / \tilde{t}-5 / 6 \tilde{t}}{2 / \tilde{t}} \\
& =\frac{1}{24} .
\end{aligned}
$$

Notice that we made use of the explicit distribution of the minimum of conditional Brownian motion again. From this last estimate, we conclude that

$$
\begin{aligned}
E & \left\{\mathrm{P}\left[\inf _{t>0} \widetilde{X}(t)>x\right]\right\} \\
& \geqq \frac{1}{24} E\left\{\mathrm{P}\left[\frac{1}{3} \leqq \inf _{t \leqq \tilde{t}}(t \widetilde{X}(t)) ; \quad \frac{1}{\widetilde{t}} \leqq \widetilde{X}(\widetilde{t}) \leqq \frac{2}{\widetilde{t}}\right]\right\} .
\end{aligned}
$$


We introduce again the "stretched" time variable $\tau=t^{3}$ and the process $\widetilde{Y}(\tau)=$ $t \widetilde{X}(t)$. The last expectation value in (24) is equal to

$$
\operatorname{Pr} .\left\{\frac{1}{3} \leqq \inf _{\tau^{\prime} \leqq \tau} \widetilde{Y}\left(\tau^{\prime}\right) ; 1 \leqq \widetilde{Y}(\tau) \leqq 2\right\}
$$

with

$$
\tau=\tilde{t}^{3}=(1 / 3 x)^{3}
$$

To estimate this probability, we proceed as follows:

$$
\begin{aligned}
\operatorname{Pr} . & \left\{1 / 3 \leqq \inf _{\tau^{\prime} \leqq \tau} \widetilde{Y}\left(\tau^{\prime}\right) ; 1 \leqq \widetilde{Y}(\tau) \leqq 2\right\} \\
& \geqq E\left\{u_{0}<u\left(x_{0}\right)<2 u_{0} ; \mathrm{P}\left[\frac{1}{3}<\inf _{\tau^{\prime} \leqq \tau} \widetilde{Y}(\tau) ; 1 \leqq \widetilde{Y}(\tau) \leqq 2\right]\right\},
\end{aligned}
$$

where $P\{\cdot\}=$ conditional probability given $u\left(x_{0}\right)$. Applying Lemma 2 (with $u\left(x_{0}\right.$ ) instead of $u(0)$ ), we have

$$
\begin{aligned}
\operatorname{Pr} . & \left\{\frac{1}{3} \leqq \inf _{\tau^{\prime}<\tau} \widetilde{Y}(\tau) ; 1 \leqq \widetilde{Y}(\tau) \leqq 2\right\} \\
& \geqq \exp \left\{-C_{0} / \tau\right\} \cdot \operatorname{Pr} .\left\{u_{0}<u\left(x_{0}\right)<2 u_{0}\right\},
\end{aligned}
$$

for some numerical constant $C_{0}=C_{0}\left(u_{0}\right)$ and all $\tau$ sufficiently small. Clearly, $\operatorname{Pr} .\left\{u_{0}<u\left(x_{0}\right)<2 u_{0}\right\} \neq 0$ for all $u_{0}$. Therefore,

$$
\operatorname{Pr} .\left\{\inf _{t^{\prime}>0} \tilde{X}\left(t^{\prime}\right)>x\right\} \geqq \exp \left\{-C^{\prime} / \tau\right\}, \tau \leqq 1,
$$

for a suitable constant $C^{\prime}$. Changing back to the variable $x$, according to (26), we conclude that

$$
\begin{aligned}
\operatorname{Pr} .\{\delta x>x\} & =\operatorname{Pr} .\left\{\inf _{t>0} \tilde{X}(t)>x\right\} \\
& \geqq e^{-27 C^{\prime} x^{3}} .
\end{aligned}
$$

This completes the proof of the lower bound for the tail probabilities for the length of rarefaction intervals.

Taking into account the upper bounds for the probabilities established in Theorem 3 of [1], the proof of Theorem 1 is complete.

\section{Appendix: Proof of Lemma 2}

Our goal here is to give a proof of the estimate of Lemma 2, namely

$$
\mathrm{P} \equiv \mathrm{P}\left\{\inf _{\tau^{\prime}<\tau} \tilde{Y}\left(\tau^{\prime}\right)>a ; b<\tilde{Y}(\tau)<c\right\} \geqq \exp \left\{-C_{0} / \tau\right\}
$$


for $\tau \leqq T_{0}$, where $\widetilde{Y}(\tau)$ is the process

$$
Y(\tau)=\beta(\tau)-u \tau^{1 / 3}+1 / 2
$$

conditioned on never hitting $Y=0$. The constants $a, b$ and $c$ satisfy

$$
0<a<1 / 2<b<c .
$$

The constant $u$ in (A.2) represents the value of $u(0)$ or $u\left(x_{0}\right)$ and is assumed to satisfy the inequality $u_{0}<u<2 u_{0}$, for some positive constant $u_{0}$; see the statement of Lemma 2.

Let $\tau_{0}$ denote the first time that the unconditioned process $Y(\tau)$ hits $Y=0$. Then, by definition, we have

$$
\mathrm{P}=\int_{b}^{c} \mathrm{P}\left\{\inf _{\tau^{\prime}<\tau} \tilde{Y}\left(\tau^{\prime}\right)>a ; \widetilde{Y}(\tau)=y\right\} d y,
$$

where

$$
\begin{aligned}
& \mathrm{P}\left\{\inf _{\tau^{\prime}<\tau} \tilde{Y}\left(\tau^{\prime}\right)>a ; \tilde{Y}(\tau)=y\right\} \\
& =\lim _{T \rightarrow \infty} \frac{\mathrm{P}\left\{\inf _{\tau^{\prime}<\tau} Y\left(\tau^{\prime}\right)>a ; Y(\tau)=y ; \tau_{0}>T\right\}}{\mathrm{P}\left\{\tau_{0}>T\right\}} \\
& =\mathrm{P}\left\{\inf _{\tau^{\prime}<\tau} Y\left(\tau^{\prime}\right)>a ; Y(\tau)=y ;\right\} \times \lim _{T \rightarrow \infty} \frac{\mathrm{P}\left\{\tau_{0}>T \mid Y(\tau)=y\right\}}{\mathrm{P}\left\{\tau_{0}>T\right\}} \\
& =\mathrm{P}\left\{\inf _{\tau^{\prime}<\tau} Y\left(\tau^{\prime}\right)>a ; Y(\tau)=y\right\} \times Q(1 / 2,0 ; y, \tau),
\end{aligned}
$$

with

$$
Q(x, 0 ; y, \tau)=\lim _{T \rightarrow \infty} \frac{\mathrm{P}\left\{\tau_{0}>T \mid Y(\tau)=y\right\}}{\mathrm{P}\left\{\tau_{0}>T \mid Y(0)=x\right\}} .
$$

In this last equation, we emphasize the dependence of the probabilities on the starting point/time.

We claim that if $y$ and $u$ are strictly positive and bounded, (i.e. $0<C<y, u<$ $C^{\prime}$ and $\left.\tau \leqq C^{\prime}\right)$, then $Q(1 / 2,0 ; y, \tau)$ is bounded from below by a positive constant, $C^{\prime \prime}$, depending only on $C$ and $C^{\prime}$, the latter bound holding uniformly in $y, \tau$, and $u$. This claim obviously implies that the probability of interest, $\mathrm{P}$, is comparable to

$$
\mathrm{P}\left\{\inf _{\tau^{\prime}<\tau} Y\left(\tau^{\prime}\right)>a ; b<Y(\tau)<c\right\} .
$$

In this way, the problem reduces to the study of the unconditional process $Y(\tau)$, which is easier to analyze.

To prove our claim, we shall evaluate the quantity $Q(1 / 2,0 ; y, \tau)$ in terms of path-integrals corresponding to Brownian motion. For notational convenience, we denote by $\mathrm{P}_{y, \tau}^{Y}$ the probability in the numerator of (A.3) and use a similar notation 
throughout. As a first step, note that

$$
\mathbf{P}_{y, \tau}^{Y}\left\{\tau_{0}>T\right\}=\int_{0}^{\infty} \mathbf{P}_{y, \tau}^{Y}\left\{\tau_{0}>1 ; Y(1)=z\right\} \mathbf{P}_{z, 1}^{Y}\left\{\tau_{0}>T\right\} d z
$$

and

$$
\mathrm{P}_{1 / 2,0}^{Y}\left\{\tau_{0}>T\right\}=\int_{0}^{\infty} \mathrm{P}_{1 / 2,0}^{Y}\left\{\tau_{0}>1 ; Y(1)=z\right\} \mathrm{P}_{z, 1}^{Y}\left\{\tau_{0}>T\right\} d z
$$

We have assumed, without loss of generality, that $\tau<1$. By Girsanov's Theorem, the probability measures induced by $Y$ and $\beta$ (standard Brownian motion) are mutually absolutely continuous. Moreover, with the obvious notation,

$$
\mathrm{P}_{z, 1}^{Y}\left\{\tau_{0}>T\right\}=E_{z, 1}^{\beta}\left\{\tau_{0}>T ; M[1, T, u, \beta]\right\},
$$

where $M[1, T, u, \beta]$ is the Radon-Nikodym derivative of $\mathrm{P}^{Y}$ with respect to $\mathrm{P}^{\beta}$, the standard Brownian motion measure, given by

$$
M[1, T, u, \beta]=\exp \left\{\int_{1}^{T} b(s) d \beta(s)-\frac{1}{2} \int_{1}^{T}(b(s))^{2} d s\right\} .
$$

Here,

$$
b(s)=-\frac{1}{3} u \tau^{-2 / 3}
$$

is the drift of $Y(\tau)$. Note that this drift is square-integrable on the interval $[1,+\infty)$, and that $b<0$ if $u>0$.

Furthermore,

$$
\begin{aligned}
& E_{z, 1}^{\beta}\left\{\tau_{0}>T ; M[1, T, u, \beta]\right\} \\
& \quad=\frac{E_{z, 1}^{\beta}\left\{\tau_{0}>T ; M[1, T, u, \beta]\right\}}{\mathrm{P}_{z, 1}^{\beta}\left\{\tau_{0}>T\right\}} \times \mathrm{P}_{z, 1}^{\beta}\left\{\tau_{0}>T\right\} \\
& \quad \cong E_{z, 1}^{\tilde{\beta}}\{M[1,+\infty, u, \tilde{\beta}]\} \times \mathrm{P}_{z, 1}^{\beta}\left\{\tau_{0}>T\right\}
\end{aligned}
$$

as $T \rightarrow+\infty$. Here, $\tilde{\beta}$ is Brownian motion conditioned on not hitting 0 , i.e., $\tilde{\beta} \approx$ Bessel-3 process (cf. [11] and Sect. 4. in [1]), and

$$
M[1,+\infty, u, \tilde{\beta}]=\exp \left\{\int_{1}^{+\infty} b(s) d \tilde{\beta}(s)-\frac{1}{2} \int_{1}^{+\infty}(b(s))^{2} d s\right\} .
$$

It can be checked by direct computation that, if $u>0$, then

$$
e^{-C\left(u^{2}+u\right)} \leqq E_{z, 1}^{\tilde{\beta}}(M[1,+\infty, u, \tilde{\beta}]) \leqq 1,
$$

for some numerical constant $\mathrm{C}$. Furthermore, the probability $\mathrm{P}_{z, 1}^{\beta}\left(\tau_{0}>T\right)$ converges to zero as $T \rightarrow+\infty$. Its asymptotic behavior is given by

$$
\mathrm{P}_{z, 1}^{\beta}\left(\tau_{0}>T\right) \propto \frac{2 z}{\sqrt{2 \pi T}}, \quad T \gg 1
$$


Putting together (A.4), (A.5) and (A.7), we arrive at an expression for $Q(1 / 2,0 ; y, \tau)$, namely

$$
\begin{aligned}
& Q(1 / 2,0 ; y, \tau) \\
& =\frac{\int_{0}^{\infty} \mathrm{P}_{y, \tau}^{Y}\left\{\tau_{0}>1 ; Y(1)=z\right\} E_{z, 1}^{\tilde{\beta}}\{M[1,+\infty, u, \tilde{\beta}]\} z d z}{\int_{0}^{\infty} \mathrm{P}_{1 / 2,0}^{Y}\left\{\tau_{0}>1 ; Y(1)=z\right\} E_{z, 1}^{\tilde{\beta}}\{M[1,+\infty, u, \tilde{\beta}]\} z d z} .
\end{aligned}
$$

For $y$ such that $b<y<c$, for $u$ such that $u_{0}<u<2 u_{0}$ and for $\tau<1$, this quantity is uniformly bounded from above and below. This follows from the bounds in (A.6). We have therefore reduced the proof of Lemma 2 to the estimation of the probability for the unconditional process $Y$, viz., $\mathrm{P}\left\{\inf _{\tau^{\prime}<\tau} Y\left(\tau^{\prime}\right)>a ; b<Y(\tau)<\right.$ c).

We will show that, given $u_{0}>0$, there exist constants $C_{0}$ and $T_{0}$, which depend on $a, b, c$ and $u_{0}$, but not on $\tau$ or $u$, such that

$$
\mathbf{P}\left\{\inf _{\tau^{\prime}<\tau} Y\left(\tau^{\prime}\right)>a ; b<Y(\tau)<c\right\} \geqq \exp \left\{-C_{0} / \tau\right\},
$$

for all $\tau \leqq T_{0}$.

For this purpose, given $a, b, c$, and $u_{0}$, select $T_{0}$ in such a way that

$$
a^{\prime} \equiv a-1 / 2+2 u_{0} T_{0}^{1 / 3}<0
$$

and

$$
b^{\prime} \equiv b-1 / 2+2 u_{0} T_{0}^{1 / 3}<c-1 / 2 \equiv c^{\prime} .
$$

With these values for $a^{\prime}$ and $b^{\prime}$, the inequalities

$$
\inf _{\tau^{\prime} \leqq T_{0}} \beta\left(\tau^{\prime}\right)>a^{\prime},
$$

and

$$
b^{\prime}<\beta(\tau)<c^{\prime}, \quad \text { for } \tau \leqq T_{0},
$$

imply that

$$
\inf _{\tau^{\prime} \leqq T_{0}} Y\left(\tau^{\prime}\right)>a,
$$

and

$$
b<Y(\tau)<c, \quad \text { for } \tau \leqq T_{0} .
$$

Therefore, if $\tau \leqq T_{0}$, then

$$
\mathrm{P} \geqq \operatorname{Prob} .\left\{\inf _{\tau^{\prime} \leqq \tau} \beta\left(\tau^{\prime}\right)>a^{\prime} ; b^{\prime}<\beta(\tau)<c^{\prime}\right\} .
$$

The latter probability is easy to estimate: since $a^{\prime}<0$, the constraint on $\inf \beta$ is irrelevant as $\tau \rightarrow 0$. Hence, for small enough $T_{0}$, we have

$$
\mathrm{P} \geqq \frac{1}{2} \operatorname{Prob} .\left\{b^{\prime}<\beta(\tau)<c^{\prime}\right\} .
$$


By direct calculation with the Gaussian distribution, we conclude that

$$
\mathrm{P} \geqq e^{-C_{0} / \tau}
$$

for some numerical constant $C_{0}=C_{0}\left(b^{\prime}, c^{\prime}\right)$.

This completes the proof of Lemma 2 .

Acknowledgement. The author thanks Dr. Weinan E for his valuable contributions that led to this sequel to our previous work. This work was supported by grants from the National Science Foundation (NSF-DMS-9207085), U.S. Army (ARO-DAAL-03-92-G0011) and U.S. Air Force (AFOSR-90-0090).

\section{References}

1. Avellaneda, M., Weinan E.: Statistical Properties of Shocks in Burgers Turbulence. Commun. Math. Phys.

2. Burgers, J.M.: The nonlinear diffusion equation. Dordrecht: Reidel, 1974

3. Hopf, E.: The partial differential equation $u_{t}+u u_{\mathrm{r}}=\mu u_{x x}$. Comm. Pure Appl. Math. 3, 201$230(1950)$

4. Gurbatov, S., Malakhov, A., Saichev, A.: Nonlinear random waves and turbulence in nondispersive media: Waves, rays and particles. New York: Manchester University Press, 1991

5. Lax, P.D.: Hyperbolic Systems of Conservation Laws and the Mathematical Theory of Shock Waves. CMBS Regional Conference Series in Applied Mathematics, 11, Society for Industrial and Applied Mathematics, Philadelphia, (1973)

6. Kida, S.: Asymptotic properties of Burgers turbulence. J. Fluid Mechanics 79, 337-377 (1977)

7. She, Z.-S., Aurell, E., Frisch, U.: The inviscid Burgers equation with initial data of Brownian type. Commun. Math. Phys. 148, 623-641 (1992)

8. Sinai, Ya.: Statistics of shocks in solutions of inviscid Burgers equation. Commun. Math. Phys. 148, $640(1992)$

9. Groenboom, P.: The concave majorant of Brownian motion. Annals of Probability, 11, No. 4. 1016-1027 (1983)

10. Pitman, J.M.: Seminar on Stochastic Processes. Cinlar, E., Chung, K.L., Getoor, R.K. (eds) Basel: Birkhauser, 1982 pp. 219-227

11. Williams, D.: Path decomposition and continuity of local time for one-dimensional diffusions. I. Proc. London Math. Society 3, 28, 738-768 (1974)

12. Vergassola, M., Dubrulle, B., Frisch, U., Noullez, A.: Burgers' equation, Devil's staircases and the mass distribution for large-scale structures, Astron. Astrophys. 289, 325-356 (1994) 
